\title{
Loss of the bloom syndrome helicase increases DNA ligase 4-independent genome rearrangements and tumorigenesis in aging Drosophila
}

Ana Maria Garcia', Robert N Salomon ${ }^{2}$, Alice Witsell ${ }^{3}$, Justine Liepkalns ${ }^{3 \dagger}$, R Brent Calder ${ }^{4}$, Moonsook Lee ${ }^{4}$, Martha Lundell ${ }^{* *}$, Jan Vijg ${ }^{4^{*}}$ and Mitch McVey ${ }^{3^{*}}$

\begin{abstract}
Background: The BLM DNA helicase plays a vital role in maintaining genome stability. Mutations in $B L M$ cause Bloom syndrome, a rare disorder associated with cancer predisposition and premature aging. Humans and mice with b/m mutations have increased frequencies of spontaneous mutagenesis, but the molecular basis of this increase is not well understood. In addition, the effect of aging on spontaneous mutagenesis in b/m mutants has not been characterized. To address this, we used a lacZ reporter system in wild-type and several mutant strains of Drosophila melanogaster to analyze mechanisms of mutagenesis throughout their lifespan.

Results: Our data show that Drosophila lacking BLM have an elevated frequency of spontaneous genome rearrangements that increases with age. Although in normal flies most genome rearrangements occur through DNA ligase 4-dependent classical end joining, most rearrangements that accumulate during aging in b/m mutants do not require DNA ligase 4, suggesting the influence of an alternative end-joining mechanism. Adult blm mutants also display reduced lifespan and ligase 4-independent enhanced tumorigenesis in mitotically active tissues.

Conclusions: These results suggest that Drosophila BLM suppresses error-prone alternative end-joining repair of DNA double-strand breaks that can result in genome instability and tumor formation during aging. In addition, since loss of BLM significantly affects lifespan and tumorigenesis, the data provide a link between error-prone end joining, genome rearrangements, and tumor formation in a model metazoan.
\end{abstract}

\section{Background}

Bloom syndrome is a rare, autosomal recessive disorder whose most striking characteristic is a predisposition to all types of cancers (reviewed in [1,2]). It is caused by mutations in the $B L M$ gene, a member of the RecQ family of DNA helicases [3]. Cells derived from Bloom syndrome patients and Blm hypomorphic mice exhibit greatly elevated genome instability, including a

\footnotetext{
* Correspondence: Martha.Lundell@utsa.edu; jan.vijg@einstein.yu.edu; mitch. mcvey@tufts.edu

+ Contributed equally

'Department of Biology, University of Texas at San Antonio, One UTSA Circle, San Antonio, TX 78249, USA

${ }^{3}$ Department of Biology, Tufts University, 165 Packard Avenue, Medford, MA 02155, USA

Full list of author information is available at the end of the article
}

dramatically increased frequency of sister chromatid exchanges $[4,5]$.

Cells with defective BLM also have a heightened mutation frequency that is partially independent of the increase in sister chromatid exchanges [6-9]. The cause of this is poorly understood, but it may be related to the increased number of chromosome aberrations and translocations that are observed in BLM mutant cells. Because these types of mutations are hypothesized to be driving forces in the development and progression of cancer in Bloom syndrome patients, understanding their origin is important.

The mus309 gene encodes the Drosophila melanogaster BLM ortholog DmBlm [10]. Flies lacking DmBlm phenocopy many of the characteristics of human Bloom syndrome, including reduced fertility and increased

\section{Biomed Central}


mitotic sister chromatid exchanges [10-12]. DmBlm is required for accurate homologous recombination (HR) repair of site-specific DNA double-strand breaks (DSBs) and for prevention of mitotic crossovers [13,14]. In its absence, DSB repair frequently proceeds through nonconservative, deletion-prone repair mechanisms. Although initiation of homologous recombination is required for the deletions observed in blm mutants [15], the molecular mechanisms responsible for deletion formation remain unknown.

Here, we report that spontaneous mutagenesis at a lacZ reporter locus is increased in Drosophila blm mutants, similar to what is observed in human Bloom syndrome patients. The increase persists through the adult lifespan and is largely due to elevated genome rearrangements, almost $50 \%$ of which involve repetitive genomic regions. Interestingly, although spontaneous rearrangements in wild-type flies depend largely on DNA ligase 4 (Lig4)-dependent classical end joining, rearrangements in blm mutants are mostly Lig4-independent. Flies lacking DmBlm, but not Lig4, also have increased tumorigenesis, suggesting that, in the absence of Blm, alternative end joining may be involved in the formation of genome rearrangements that can drive tumorigenesis.

\section{Results and discussion}

\section{Genome rearrangements involving repeated sequences are common in blm mutants}

To characterize the consequences of DmBlm deficiency on spontaneous mutagenesis, we crossed a null mus309 mutation [12] into a $w^{1118}$ background harboring a lacZ reporter construct that allows us to measure somatic mutation frequencies and mutation spectra [16] (Additional file 1). For these studies, we used a lacZ reporter integrated at cytological position $87 \mathrm{E}$ on chromosome 3, which exhibits a spontaneous mutation frequency nearly identical to the average obtained using multiple other insertion sites [17]. The blm mutant stocks containing the reporter construct were isogenized and homozygous mutants were recovered at days 1, 14, and 28 after adult eclosion. Plasmid rescue was performed and mutation frequencies were calculated on a per locus basis as the number of mutant lac $Z$ copies versus the total number of copies rescued from a given amount of DNA (Additional file 2).

We observed a significantly higher mutation frequency for blm mutants relative to wild-type at all ages (Figure 1a; $P<0.001$ for both sexes, one-way ANOVA). As found previously, mutation frequencies were slightly higher in females than in males [17]. This difference is largely due to an increased frequency of point mutations in females (see below). Since point mutations often result from

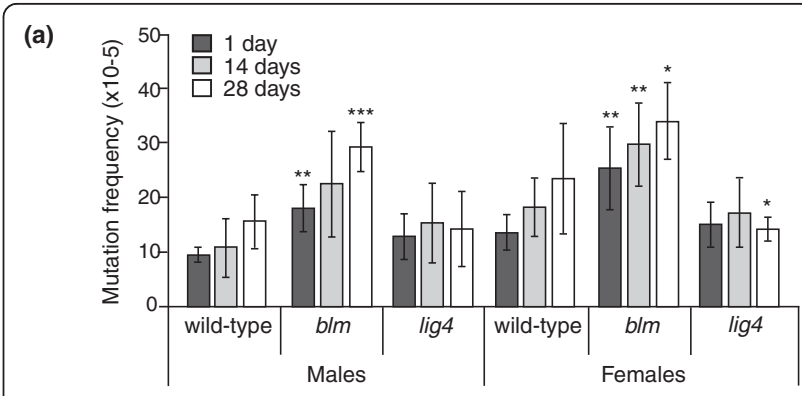

(b)

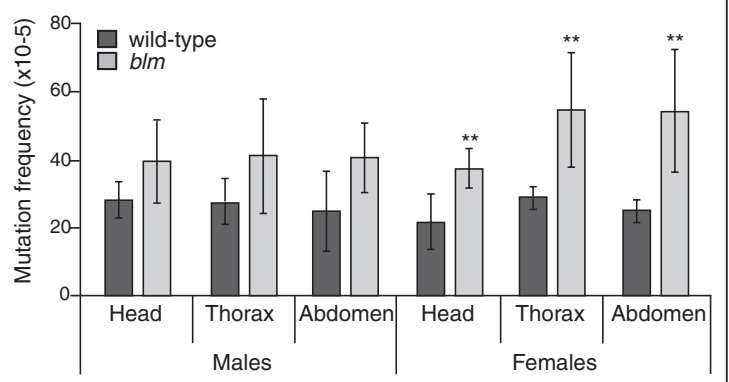

(c)

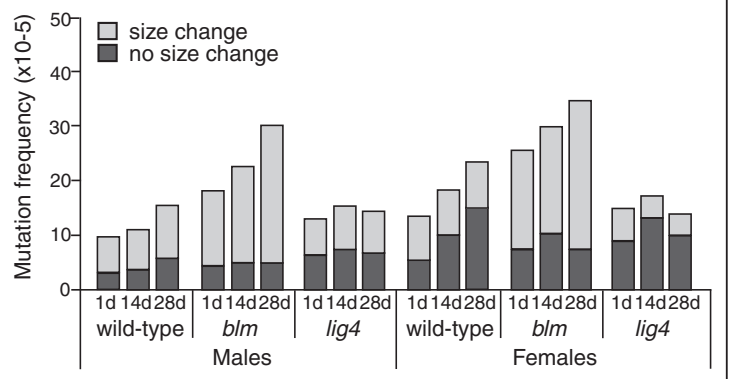

Figure 1 Somatic mutation frequency and percentage of genome rearrangements increases in blm mutants with age. (a) Somatic mutation frequencies grouped by sex and age. Each bar represents five to seven independent measurements. Error bars represent standard deviations. ${ }^{*} P<0.05$ compared to wild type, ${ }^{* *} P$ $<0.01$, ***P $<0.001$ (one-way ANOVA). (b) Somatic mutation frequencies in various body regions. Samples of head, thorax, and abdominal tissue were collected from 35-day-old male and female flies and somatic mutation frequencies were determined. Each bar represents three to six independent measurements. Error bars represent standard deviations. ${ }^{*} P \leq 0.01$ compared to wild type (two-tailed unpaired t-test). (c) Somatic mutation frequencies that involved either a size change or no size change, grouped by sex and age. Size change mutations correspond to genome rearrangements or lacZ internal deletions, while no size change mutations are usually point mutations.

replication errors, females, which have a larger body size, may have more opportunities to accumulate point mutations.

The increased mutation frequency in blm mutants was not specific to a particular region of the fly, but was observed in head, thorax, and abdominal tissues (Figure 1b). Although the differences in tissue-specific mutation frequencies between blm mutant and wild-type males 
were less significant compared to those observed using whole flies, this is likely a consequence of a higher mutation frequency in the wild-type males in this particular experiment (data not shown).

To examine the types of mutations that occurred in blm mutants, we performed restriction analysis on a subset of plasmids. Plasmids that show no size change after digestion with the AvaI restriction enzyme generally harbor point mutations, while plasmids showing a size change after digestion involve rearrangements with one breakpoint in the lacZ gene and the other elsewhere in the fly genome. We have previously shown that genome rearrangements, including deletions and both intraand inter-chromosomal translocations, are the predominant type of mutation in flies [17]. Interestingly, the increased mutation accumulation in blm mutants appeared to be almost entirely due to an increase in genome rearrangements (Figure 1c). This increase was evident in both males and females $(P<0.001$, Fisher's exact test).

To further characterize the genome rearrangements recovered from the $b l m$ mutants, we sequenced the lac $Z$ gene in a representative number of mutants and identified the breakpoints. Using BLAST alignment of the recovered sequence against the Drosophila genome, we definitively mapped the breakpoints for 11 out of 21 rearrangements (Additional file 3). Three rearrangements were deletions in the lac $Z$ transgene, while eight were larger deletions or inversions with the breakpoints outside the reporter gene on the same chromosome. Surprisingly, we were unable to map the breakpoints for $48 \%$ of the rearrangements isolated from $\mathrm{blm}$ mutants because one side of the breakpoint was located in highly repetitive sequences. Six of these sequences corresponded to various transposons, including accord\{\}818, stalker, and copia, while four matched repetitive sequences that are highly represented in both euchromatic and heterochromatic regions of the fly genome. Previous studies using the same lacZ reporter found a much lower proportion of genome rearrangements involving repetitive sequences (6 out of 55 , or $11 \%, P=0.0003$ compared to blm mutants, two-tailed Fisher's exact test) $[17,18]$. Therefore, the increased somatic mutation frequency in $\mathrm{blm}$ mutants is due, at least in part, to an increased frequency of genome rearrangements involving highly repetitive sequences.

\section{Differential requirements for rearrangement formation in wild type and blm mutants}

The simplest model to explain the observed genome rearrangements involves inaccurate repair of two simultaneous DSBs. It is possible that recombination between two breaks could result in deletions and translocations and that DmBlm could suppress these types of rearrangements by preventing homeologous recombination between closely related sequences [19]. However, careful inspection of the junction sequences at the sites of the genome rearrangements isolated from wild type [18] and $b l m$ mutants did not reveal extensive regions of homology. To determine if classical non-homologous end joining (C-NHEJ) might be required for the formation of these rearrangements, we repeated our analysis in flies lacking DNA ligase 4, which is required for CNHEJ $[20,21]$. Surprisingly, we saw no age-dependent increase in mutation frequency in lig4 mutants (Figure 1a). In addition, the percentage of genome rearrangements in lig4 mutants was significantly less compared to wild-type flies (Figure 1c; $P \leq 0.01$ for both sexes at all ages, two-tailed Fisher's exact test), indicating that Lig4dependent C-NHEJ is responsible for most of the genome rearrangements that accumulate during normal development and aging.

Previous studies from our lab and others have demonstrated that repair of site-specific DSBs in blm mutants is frequently accompanied by large deletions $[14,15]$, a feature characteristic of Lig4-independent alternative end joining $[22,23]$. We therefore wished to determine if the genome rearrangements that occur in blm mutants rely on classical or alternative end joining. We constructed lig4 blm double mutants containing the lac Z reporter construct and measured the mutation frequency and spectra in 28-day-old flies. Spontaneous mutagenesis frequencies for the lig4 blm flies were similar to $b l m$ single mutants, but were significantly different from lig4 mutants (Figure 2a; $P=0.029$ for males, $P$ $<0.001$ for females, one-way ANOVA). Similarly, the majority of mutations in lig4 blm flies were genome rearrangements, paralleling the blm mutant phenotype (Figure 2b). In contrast to wild-type flies, therefore, most of the genome rearrangements that occur in flies lacking the DmBlm helicase do not require DNA ligase 4 and are instead generated through an alternative endjoining mechanism.

\section{Increased tumor formation and reduced lifespan in blm mutant flies}

One hallmark of Bloom syndrome is cancer predisposition at an early age [24]. Recently, several groups have reported that adult flies develop dysplasia and tumors in mitotically active tissues, including intestinal and germline tissues $[25,26]$. These tumors arise more frequently in older flies [27]. We reasoned that the increased frequency of genome rearrangements observed in the absence of DmBlm might promote tumorigenesis. To test this, we performed histopathological staining on sectioned tissues of wild-type and blm mutant flies at 35,50 , and 60 days of age. Representative histological 


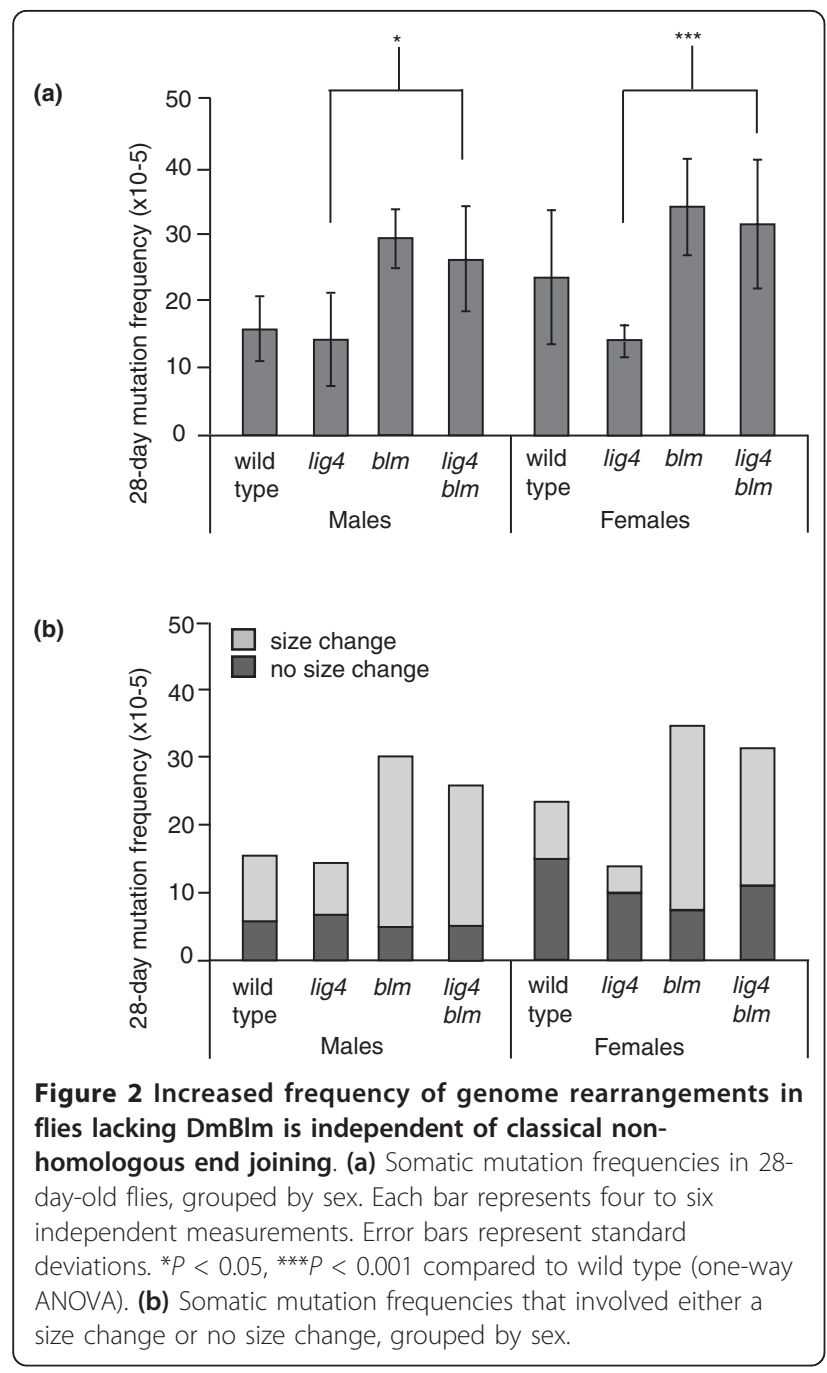

sections from 35-day-old control and age-matched blm mutant flies are shown in Figure 3. Normal fly tissues (Figure 3a, c, e) are characterized by a high degree of cytological regularity and orderliness of tissue architecture. In contrast, tumors (Figure $3 \mathrm{~b}, \mathrm{~d}, \mathrm{f}$ ) are composed of densely crowded masses of irregularly arrayed tumor cells. Although no metastases to distant tissues were observed, the gut tumors were frequently observed to partially or fully occlude the intestinal lumen. Germline tumors consisted of masses of proliferating tumor cells that were morphologically similar to stem cells. Like many mammalian tumor cells, individual fly tumor cells were variable in size and shape and showed high nucleus to cytoplasm ratios.

Similar to Bloom syndrome patients, flies lacking DmBlm had an increased frequency of tumor development at early ages. We observed a significant difference in overall tumor formation between 35-day-old wild-

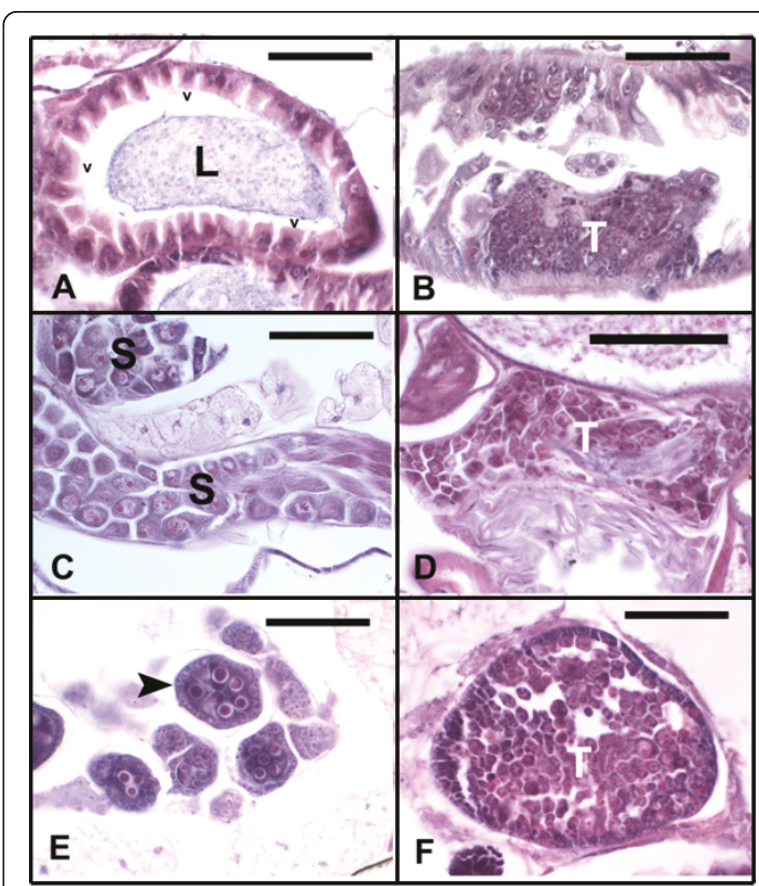

G

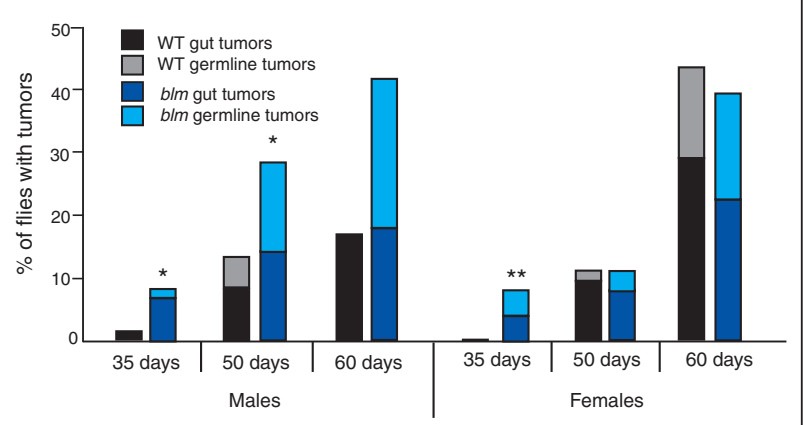

Figure $3 \mathrm{blm}$ mutant adults are predisposed to early tumor development. (a) Transverse section through midgut of wild-type fly. Normal, uniform-appearing villi (V) protrude into the gut lumen (L). A cluster of bacteria and yeasts (normal gut flora) is present within the lumen. (b) Tumor within the midgut of a blm mutant fly. A dense cluster of small tumor cells (T) arises from within the mucosa and forms an irregular mass that protrudes into the gut lumen. (c) Normal testis from a wild-type fly. Normal spermatocytes (S) in various stages of development are present. (d) Tumor within the testis of a blm mutant fly. Large numbers of small tumor cells (T) resembling early germ line precursors have replaced normal spermatocytes within the testis. (e) Normal maturing ovarian follicles from a wild-type fly. The largest (arrowhead) contains normal cyst cells surrounded by a single layer of follicular cells. (f) Ovarian tumor from a blm mutant fly. Large numbers of tumor cells (T) resembling immature germ line precursor cells replace normal cystocytes. All sections were prepared from 35-day-old flies and were stained with hematoxylin and eosin. Each scale bar $=50$ microns. (g) Histogram showing tumor frequency in 35- to 60-dayold adult flies. ${ }^{*} P<0.05$ compared to wild type (WT; two-tailed Fisher's exact test), ${ }^{* *} P<0.01$. 
type and $b l m$ mutants (Figure $3 g ; P=0.05$ and $P=$ 0.0087 for males and females, respectively). Older males also had increased tumorigenesis relative to wild type ( $P$ $=0.0047$ at 50 days), but tumor frequency was similar in older wild-type and $b l m$ mutant females. Germline tumors, which are rare in wild-type flies, comprised a significant fraction of tumors in blm mutants, particularly in males. This could indicate that certain cell populations within the testes of $b l m$ males may be more prone to neoplastic transformation. Interestingly, there was no significant difference in tumor frequency between wild type and lig4 mutants (Additional file 4), suggesting that increased tumorigenesis is not a result of loss of DSB repair capacity in general. Overall, these results agree with previous findings that tumors accumulate with age in Drosophila [27] and indicate that tumorigenesis in flies can be influenced by the status of tumor suppressor genes like $B L M$.

In Drosophila, DSB repair relies more heavily on HR as flies age [28]. Because DmBlm is important for successful completion of HR and plays an important role in the prevention of spontaneous genome rearrangements, we hypothesized that it might also act to promote longevity. To test this, we performed lifespan analysis on isogenic wild-type, lig4, and blm mutant males and females. Loss of DmBlm significantly shortened mean and maximum lifespan in both sexes $(P<0.0001$, Wilcoxon rank-sum test; Figure 4). The survivorship plots of the blm mutants diverged from the wild-type plots at approximately 20 days, and the mean lifespan of blm mutants was reduced more than $35 \%$ relative to wild type. In contrast, loss of Lig4 had no significant effect on either the mean or maximum lifespan of female flies $(P=0.83)$. The survivorship plot of the lig4 mutant males closely paralleled that of wild-type flies until approximately 45 days, at which point it diverged, intersecting with the blm curve near the maximum lifespan of both mutants.

We also quantified tumor formation and lifespan in the double mutants. For both males and females, tumor frequency in lig4 blm mutants was similar to levels observed in blm single mutants (Additional file 4), matching the spontaneous mutagenesis results (Figure 2). Interestingly, the mean and maximum lifespan of both males and females was further decreased in lig4 $\mathrm{blm}$ flies relative to blm single mutants (Additional file 5). Thus, C-NHEJ repair of DNA DSBs becomes important for organismal lifespan when HR repair is compromised.

\section{Conclusions}

Taken together, our results indicate that genome instability in Drosophila, specifically in the form of chromosome rearrangements, is affected to a greater extent by impairment of HR repair than by loss of C-NHEJ. Unlike genome rearrangements that occur during aging

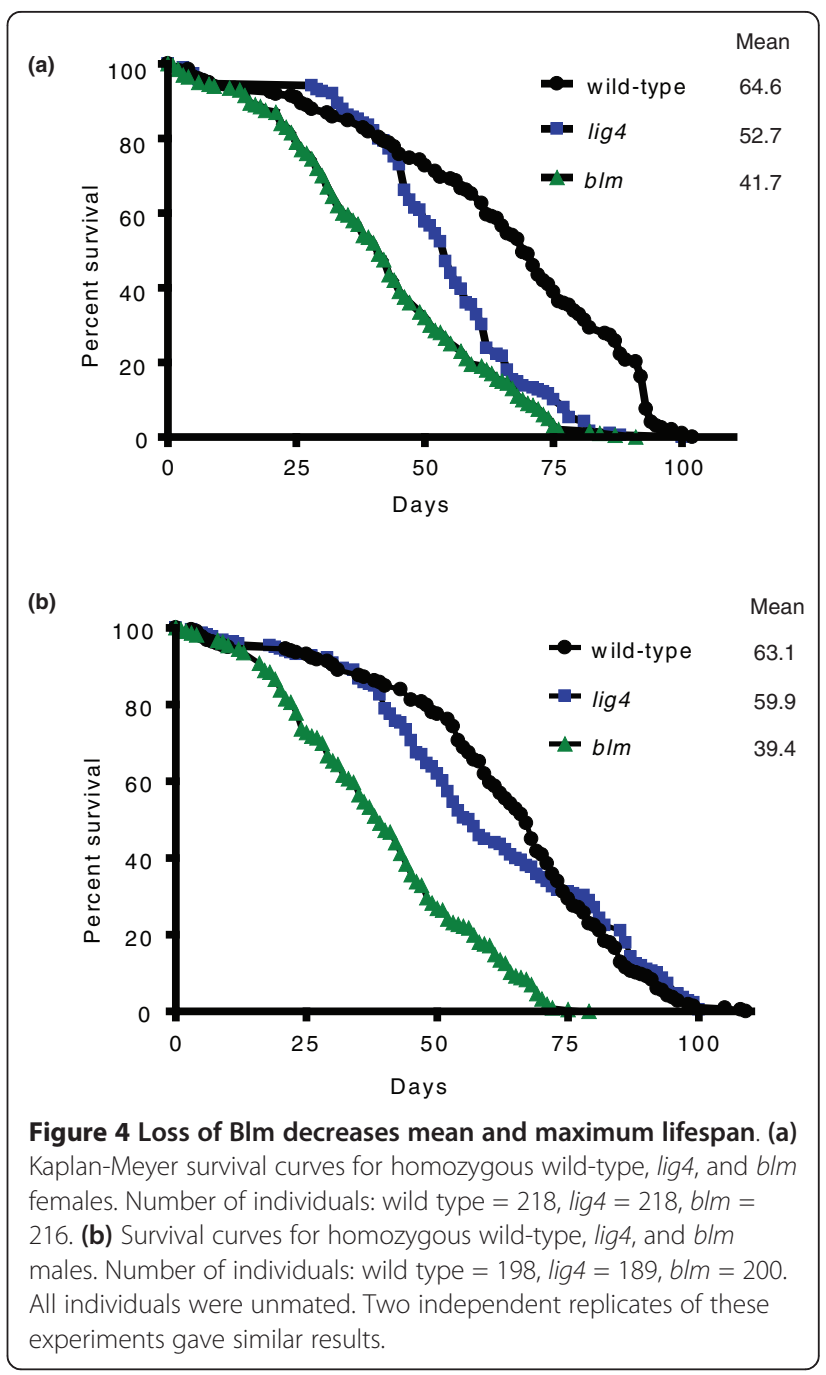

in wild-type flies, most rearrangements in blm mutants are Lig4-independent. Because blm mutants are proficient in the initial resection and strand invasion steps of HR [15], we hypothesize that DSB repair in the absence of $\mathrm{DmBlm}$ is more likely to proceed by alternative endjoining mechanisms that involve extensive resection at DNA ends. This leads to an increase in genome rearrangements and may predispose to tumorigenesis and reduced longevity. Interestingly, recent findings demonstrate that alternative end joining also plays a dominant role in translocation formation in mammals $[29,30]$. In combination with these observations, our study raises the interesting possibility that inaccurate end-joining repair may be a primary cause of pathology in Bloom syndrome and may also be a critical factor in aging.

\section{Materials and methods}

\section{Drosophila stocks and genetics}

Flies were raised on standard cornmeal agar medium. All stocks contained a lac Z reporter construct integrated 
at chromosome 3 position 87E (line 11) [17]. The lig $4^{169 a}$ mutation deletes the majority of the LIG4 locus, including the promoter [31]. The $m u s 309^{N 1}$ mutation removes the region encoding the amino terminus of DmBlm, including part of the conserved helicase domain [12]. Females from the lac $Z$ wild-type and mutant stocks were subjected to at least four backcrosses with $w^{1118}$ males to create isogenic stocks. Heterozygous lac $Z^{11}$ mus $309^{N 1} / T M 3$, lig $4^{169 a} / F M 7 w$; lac $Z^{11} / T M 3$, and lig4 ${ }^{169 a} / F M 7 w$; lac $Z^{11}{\text { mus } 309^{N 1} / T M 3}$ stocks were created by standard genetic crosses and mutations were verified by PCR.

\section{Genomic DNA isolation}

For mutation frequency studies, flies were snap frozen at various ages and stored at $-80^{\circ} \mathrm{C}$. Each sample consisted of 50 pooled male or female flies. For the tissue-specific experiments, each sample consisted of 100 heads, thoraxes, or abdomens that were isolated by cutting snap-frozen flies with a razor blade. Flies were homogenized in $600 \mu \mathrm{l}$ of lysis buffer $(10 \mathrm{mM}$ Tris- $\mathrm{HCl}, \mathrm{pH} 8.0 ; 10 \mathrm{mM}$ EDTA; $150 \mathrm{mM} \mathrm{NaCl}$ ) in $2 \mathrm{ml}$ eppendorf tubes using a battery-operated pestle. To the homogenate, $12 \mu \mathrm{l}$ of Proteinase $\mathrm{K}(25 \mathrm{mg} / \mathrm{ml}), 60 \mu \mathrm{l}$ 10\% SDS and $10 \mu \mathrm{l}$ RNase A $(20 \mu \mathrm{g} / \mathrm{ml})$ were added and samples were incubated at $65^{\circ} \mathrm{C}$ while rotating during 30 minutes. Genomic DNA was subsequently extracted from these samples using phenol/chloroform as described [16]. DNA yield and quality were estimated by electrophoresis of a small amount of the purified DNA on a 1.0\% agarose gel and by spectrophotometric measurement.

\section{Mutation analysis}

The mutation frequency was determined as described in detail elsewhere [16]. Briefly, isolated DNA from 50 flies or 100 body parts was digested for 1 hour at $37^{\circ} \mathrm{C}$ with HindIII $(40 \mathrm{U})$ in the presence of magnetic beads coated with lacI-lac Z fusion protein. The lac $Z$ plasmid was then eluted from the beads by incubation with isopropyl $\beta$-D1-thiogalactopyranoside (IPTG), circularized by ligation with T4 DNA ligase (New England Biolabs, Ipswich, MA, USA), precipitated with ethanol and used to electrotransform Escherichia coli ( $\triangle l a c Z, \Delta$ galE $\left.^{-}\right)$. Each mutant frequency determination point was based on at least three replicates from the same sample, that is, three 50-fly groups or 100 body parts from the same population, with a minimum of 100, 000 colonies for each rescue. LacZ plasmids from mutant colonies were further characterized as described in detail elsewhere [17]. Sequencing reactions of purified mutant plasmids were conducted at the Albert Einstein College of Medicine Genomics core facility. The returned chromatograms were analyzed with Sequencher (Gene Codes, Ann Arbor, MI, USA). Analysis of large rearrangements consisting of non-lacZ sequences was carried out using the fly genome database [32]. After alignment with the D. melanogaster sequence, the chromosomal origins of the flanking sequences were determined and the orientation and the type of chromosomal rearrangements deduced as described [33].

\section{Histology}

Cohorts of 20 flies were immersed in Telly's fixative (20 parts $70 \%$ ethanol, 2 parts $37 \%$ formalin, 1 part glacial acetic acid) for a minimum of 48 hours at $4^{\circ} \mathrm{C}$ prior to processing with a Leica ASP 300 automatic tissue processor using standard techniques. The processed flies were embedded in paraffin blocks, sectioned into ribbons 6 to 7 microns thick, placed onto glass slides, and baked at $65^{\circ} \mathrm{C}$ for 12 hours to increase tissue adherence prior to staining with hematoxylin and eosin. Digital microphotography was done with an Olympus VANOX-T photomicroscope and a $\mathrm{Q}$ Imaging digital camera. All samples were coded and scored without prior knowledge of genotype.

\section{Lifespan analysis}

Homozygous flies collected from isogenized stocks were aged in groups of $<20$ flies in vials kept at $25^{\circ} \mathrm{C}$ and constant humidity on a 12-hour light:12-hour dark cycle. Flies were transferred without anesthesia to new food every 2 to 3 days. For each lifespan experiment, at least 150 flies were monitored and deaths were scored every 1 to 2 days. Lifespan experiments were repeated three times for wild-type and $b l m$ flies and two times for lig4 flies and lig4 blm flies.

\section{Statistical analysis}

Somatic mutation frequencies were compared with oneway ANOVA tests followed by Tukey-Kramer multiple comparisons tests for three or more different genotypes and Mann-Whitney tests for comparisons between two genotypes. Gaussian distributions were confirmed using the Kolmogorov and Smirnov method. For tumor frequencies, two-tailed Fisher's exact tests were used to compare between genotypes. Survivorship curves were compared using Wilcoxon matched pairs signed-rank tests. All statistical analysis was conducted using GraphPad Prism software.

\section{Additional material}

Additional file 1: Methods used to calculate spontaneous mutation frequency and analyze mutations.

Additional file 2: Information about the numbers of colonies obtained in the mutation frequency experiments.

Additional file 3: Information about the genome rearrangements isolated in this study.

Additional file 4: Set of tables showing data related to tumor frequency in wild-type and mutant backgrounds and statistical analysis for tumor frequency in different genetic backgrounds. 


\section{Additional file 5: Lifespan analysis for lig4 blm double mutant flies.}

\section{Abbreviations}

C-NHEJ: classical non homologous end joining; DmBlm: Drosophila melanogaster BIm; DSB: double-strand break; HR: homologous recombination; Lig4: DNA ligase 4.

\section{Acknowledgements}

This work was supported by an Ellison Medical Foundation New Scholar in Aging Award, American Cancer Society grant RSG-11-051-01-DMC, and NSF grant MCB0643253 (to MM) and NIH grants AG020438 and RR024346 (to JV and $\mathrm{ML}$ )

\section{Author details}

'Department of Biology, University of Texas at San Antonio, One UTSA Circle, San Antonio, TX 78249, USA. ²Department of Pathology, Tufts Medical Center, 800 Washington Street, Boston, MA 02111, USA. ${ }^{3}$ Department of Biology, Tufts University, 165 Packard Avenue, Medford, MA 02155, USA. ${ }^{4}$ Department of Genetics, Albert Einstein College of Medicine, 1301 Morris Park Avenue, Bronx, NY 10461, USA.

\section{Authors' contributions}

AMG designed the study, conducted and analyzed the mutation frequency experiments and wrote the manuscript. MLu designed the study. JV and MM designed the study and wrote the manuscript. MLee conducted and analyzed the mutation frequency experiments. RS carried out the tumorigenesis studies. RBC carried out bioinformatic analysis of the rearrangements. AW and JL carried out the lifespan analysis. All authors read and approved the final manuscript for publication.

\section{Competing interests}

The authors declare that they have no competing interests.

Received: 14 September 2011 Revised: 25 November 2011

Accepted: 19 December 2011 Published: 19 December 2011

\section{References}

1. German J: Bloom's syndrome. Dermatol Clin 1995, 13:7-18

2. Monnat RJ Jr: Human RECQ helicases: roles in DNA metabolism, mutagenesis and cancer biology. Semin Cancer Biol 2010, 20:329-339.

3. Ellis NA, Groden J, Ye TZ, Straughen J, Lennon DJ, Ciocci S, Proytcheva M, German J: The Bloom's syndrome gene product is homologous to RecQ helicases. Cell 1995, 83:655-666.

4. Chaganti RS, Schonberg S, German J: A manyfold increase in sister chromatid exchanges in Bloom's syndrome lymphocytes. Proc Natl Acad Sci USA 1974, 71:4508-4512.

5. Luo G, Santoro IM, McDaniel LD, Nishijima I, Mills M, Youssoufian H, Vogel H, Schultz RA, Bradley A: Cancer predisposition caused by elevated mitotic recombination in Bloom mice. Nat Genet 2000, 26:424-429.

6. Tereshchenko IV, Chen Y, McDaniel LD, Schultz RA, Tischfield JA, Shao C Small scale genetic alterations contribute to increased mutability at the $X$-linked Hprt locus in vivo in Blm hypomorphic mice. DNA Repair (Amst) 2010, 9:551-557.

7. Warren ST, Schultz RA, Chang CC, Wade MH, Trosko JE: Elevated spontaneous mutation rate in Bloom syndrome fibroblasts. Proc Natl Acad Sci USA 1981, 78:3133-3137.

8. Langlois $R G$, Bigbee $W L$, Jensen $R H$, German J: Evidence for increased in vivo mutation and somatic recombination in Bloom's syndrome. Proc Natl Acad Sci USA 1989, 86:670-674.

9. Tachibana A, Tatsumi K, Masui T, Kato T: Large deletions at the HPRT locus associated with the mutator phenotype in a Bloom's syndrome lymphoblastoid cell line. Mol Carcinog 1996, 17:41-47.

10. Kusano K, Berres ME, Engels WR: Evolution of the RECQ family of helicases: A Drosophila homolog, Dmblm, is similar to the human Bloom syndrome gene. Genetics 1999, 151:1027-1039.

11. Kusano K, Johnson-Schlitz DM, Engels WR: Sterility of Drosophila with mutations in the Bloom syndrome gene - complementation by Ku70. Science 2001, 291:2600-2602.
12. McVey M, Andersen SL, Broze Y, Sekelsky J: Multiple functions of Drosophila BLM helicase in maintenance of genome stability. Genetics 2007, 176:1979-1992

13. Adams MD, MCVey M, Sekelsky JJ: Drosophila BLM in double-strand break repair by synthesis-dependent strand annealing. Science 2003, 299:265-267.

14. Johnson-Schlitz D, Engels WR: Template disruptions and failure of double Holliday junction dissolution during double-strand break repair in Drosophila BLM mutants. Proc Natl Acad Sci USA 2006, 103:16840-16845.

15. McVey M, Larocque JR, Adams MD, Sekelsky JJ: Formation of deletions during double-strand break repair in Drosophila DmBlm mutants occurs after strand invasion. Proc Natl Acad Sci USA 2004, 101:15694-15699.

16. Garcia AM, Busuttil RA, Rodriguez A, Cabrera C, Lundell M, Dolle ME, Vijg J: Detection and analysis of somatic mutations at a lacZ reporter locus in higher organisms: application to Mus musculus and Drosophila melanogaster. Methods Mol Biol 2007, 371:267-287.

17. Garcia AM, Derventzi A, Busuttil R, Calder RB, Perez E Jr, Chadwell L, Dolle ME, Lundell M, Vijg J: A model system for analyzing somatic mutations in Drosophila melanogaster. Nat Methods 2007, 4:401-403.

18. Garcia AM, Calder RB, Dolle ME, Lundell M, Kapahi P, Vijg J: Age- and temperature-dependent somatic mutation accumulation in Drosophila melanogaster. PLoS Genet 2010, 6:E1000950.

19. Kappeler M, Kranz E, Woolcock K, Georgiev O, Schaffner W: Drosophila bloom helicase maintains genome integrity by inhibiting recombination between divergent DNA sequences. Nucleic Acids Res 2008, 36:6907-6917.

20. Preston CR, Flores CC, Engels WR: Differential usage of alternative pathways of double-strand break repair in Drosophila. Genetics 2006, 172:1055-1068

21. Wei DS, Rong YS: A genetic screen for DNA double-strand break repair mutations in Drosophila. Genetics 2007, 177:63-77.

22. Mladenov $\mathrm{E}$, lliakis $\mathrm{G}$ : Induction and repair of DNA double strand breaks: the increasing spectrum of non-homologous end joining pathways. Mutat Res 2011, 711:61-72.

23. Yan CT, Boboila C, Souza EK, Franco S, Hickernell TR, Murphy M, Gumaste $S$, Geyer M, Zarrin AA, Manis JP, Rajewsky K, Alt FW: IgH class switching and translocations use a robust non-classical end-joining pathway. Nature 2007, 449:478-482.

24. German J: Bloom syndrome: a mendelian prototype of somatic mutational disease. Medicine 1993, 72:393-406.

25. Biteau B, Hochmuth CE, Jasper H: JNK activity in somatic stem cells causes loss of tissue homeostasis in the aging Drosophila gut. Cell Stem Cell 2008, 3:442-455.

26. Apidianakis Y, Pitsouli C, Perrimon N, Rahme L: Synergy between bacterial infection and genetic predisposition in intestinal dysplasia. Proc Natl Acad Sci USA 2009, 106:20883-20888.

27. Salomon RN, Jackson FR: Tumors of testis and midgut in aging flies. Fly (Austin) 2008, 2:265-268.

28. Preston CR, Flores C, Engels WR: Age-dependent usage of double-strandbreak repair pathways. Curr Biol 2006, 16:2009-2015.

29. Simsek $D$, Jasin M: Alternative end-joining is suppressed by the canonical NHEJ component Xrcc4-ligase IV during chromosomal translocation formation. Nat Struct Mol Biol 2010, 17:410-416.

30. Zhang $Y$, Jasin M: An essential role for CtIP in chromosomal translocation formation through an alternative end-joining pathway. Nat Struct Mol Biol 2011, 18:80-84

31. McVey M, Radut D, Sekelsky Jj: End-joining repair of double-strand breaks in Drosophila melanogaster is largely DNA ligase IV independent. Genetics 2004, 168:2067-2076

32. FlyBase. [http://flybase.org/].

33. Dolle ME, Vijg J: Genome dynamics in aging mice. Genome Res 2002, $12: 1732-1738$

doi:10.1186/gb-2011-12-12-r121

Cite this article as: Garcia et al:: Loss of the bloom syndrome helicase increases DNA ligase 4-independent genome rearrangements and tumorigenesis in aging Drosophila. Genome Biology 2011 12:R121. 